\title{
A review on Approximate Computing applied to meteorological forecast models using software-based techniques
}

\author{
Marcelo Augusto Sudo, Álvaro Luiz Fazenda \\ Instituto de Ciência e Tecnologia - Universidade Federal de São Paulo (UNIFESP) \\ São José dos Campos - SP - Brazil \\ marcelo.sudo@gmail.com, alvaro.fazenda@unifesp.br
}

\begin{abstract}
This study is part of research on Approximate Computing software-based techniques applied to meteorological forecast numerical models. Currently, a Systematic Review is ongoing, and it will serve as a reference to verify which algorithms have already been ported and which techniques are being used. This document focuses only on some approximated computing techniques, especially on mixed or reduced precision. Conclusions up to now show just a few research articles regarding the subject, so there are still many aspects to unveil.
\end{abstract}

\section{Introduction}

Approximate Computing (AC) relies on the ability of many systems and applications to tolerate some loss of quality or optimality in the computed result [Han and Orshansky 2013]. The term was first mentioned by Lim and Tillmann (1986) in the paper "End-to-End Speech Freeze-Out Fractions in a Network with Speech Interpolations". After that, many other research projects were done, initially considering energy savings for digital systems, but with the evolution of techniques, other fields were involved such as software and data. AC could be classified in different subclasses like Approximate algorithms, Approximate data, and Approximate hardware, as defined in Li et al. (2017).

The purpose of this Systematic Review is to do further analysis on the algorithm level to better understand which kind of hardware has been used to, which programming languages were most popular for $\mathrm{AC}$, and what software methods and procedures have been implemented. Besides, it is important to know which kind of numerical models have already been ported to AC. This Review is the first source for a research project intending to port parts of a weather forecast model (WFM) through AC software-based techniques. Since WFM usually demands high-performance computing facilities, which implies high energy consumption to process high precision floating point data and operations, it poses as a potential candidate to be optimized by $\mathrm{AC}$, however, the tolerance to quality loss needs to be further investigated.

\section{Methodology}

The methodology applied in this Systematic Review consisted in the elaboration of five Questions of Research listed in Table 1, and the consequent answers to them.

Table 1 - Questions of Research

\begin{tabular}{|l|l|}
\hline$\#$ & Question \\
\hline
\end{tabular}




\begin{tabular}{|c|l|}
\hline Q1 & $\begin{array}{l}\text { Which kind of Approximate Computing has been used? Hardware-based? Software- } \\
\text { based? Both? }\end{array}$ \\
\hline Q2 & Considering the researches focused only in software techniques: \\
\hline Q3 & Which kind of hardware has been used? CPU, GPU, FPGA, others? \\
\hline Q4 & $\begin{array}{l}\text { Which numerical models have already been ported to AC? (image, video, loT, machine } \\
\text { learning, a mathematical system of equations, weather forecast) }\end{array}$ \\
\hline Q5 & $\begin{array}{l}\text { Which software-based techniques of AC are being used? (loop perforation, memoization, } \\
\text { neural network, mixed/reduced precision) }\end{array}$ \\
\hline
\end{tabular}

\subsection{Digital Libraries}

The source of articles analyzed up to now is one of the main libraries used by researchers all over the world: IEEE Xplore Digital Library (https://ieeexplore.ieee.org/Xplore/home.jsp). The searches were done on March 1st of 2020, and consider both Journals and Conference articles without distinction.

For future work, ACM Digital Library (https://dl.acm.org/) will also be included in the search because of its relevance in community.

\subsection{Keywords for searching}

The keywords used in the searches were separated into five groups, according to Table 2 . For group one, the intention was to answer the first question (Q1). The second group intend to search for papers where AC is applied to weather forecast models. The search related to the third group found $\mathrm{AC}$ as some specific software-based techniques. The fourth group specifies $\mathrm{AC}$ with any software-based procedure. The last group found $\mathrm{AC}$ as a machine learning method. The number of citations found can also be viewed in Table 2.

Table 2 - Searching groups with Keywords

\begin{tabular}{|c|c|c|}
\hline Group & Keywords & Articles \\
\hline 1 & "approximate computing" OR "mixed precision" & 1278 \\
\hline 2 & $\begin{array}{c}\text { ("approximate computing" OR "mixed precision") AND ("weather } \\
\text { forecasting" OR "weather prediction" OR "climate" OR "meteorology" } \\
\text { OR "global atmosphere") }\end{array}$ & 8 \\
\hline 3 & $\begin{array}{c}\text { ("approximate computing" OR “mixed precision") AND ("precision } \\
\text { scale" OR “loop perforation" OR “memoization" OR "value } \\
\text { approximation") }\end{array}$ & 29 \\
\hline 4 & $\begin{array}{l}\text { ("approximate computing" OR “mixed precision") AND ("software" } \\
\text { OR “algorithm") AND ("techniques" OR "approaches") }\end{array}$ & 131 \\
\hline 5 & $\begin{array}{l}\text { ("approximate computing" OR “mixed precision") AND (“machine } \\
\text { learning" OR “artificial intelligence" OR "deep learning" OR "data } \\
\text { science") }\end{array}$ & 250 \\
\hline
\end{tabular}




\subsection{Results}

A total 1278 articles were found related to AC, in general, until the date of this Systematic Review. As seen in Figure 1, the amount of researches had a boost in the last five years with an average of 196.2 publications per year, considering 2015 to 2019, against an average of only 34.0 between 2011 and 2014 .

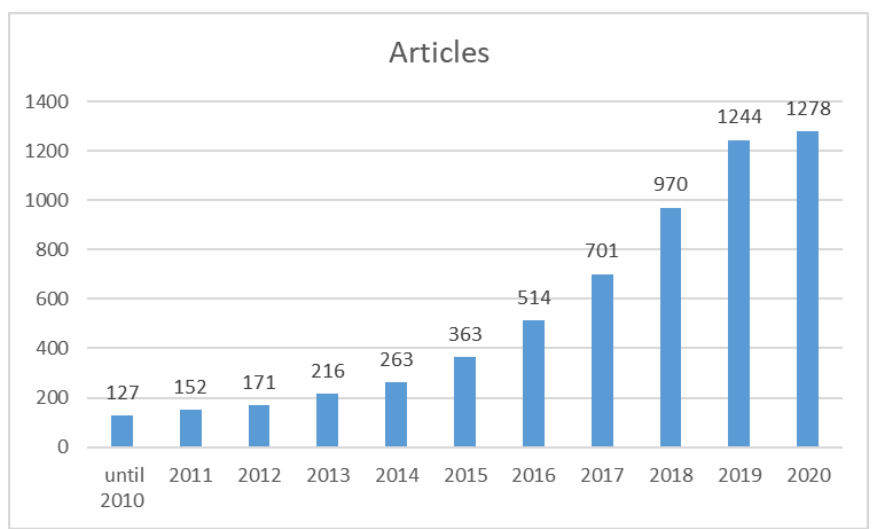

Figure 1. Approximate Computing articles Histogram

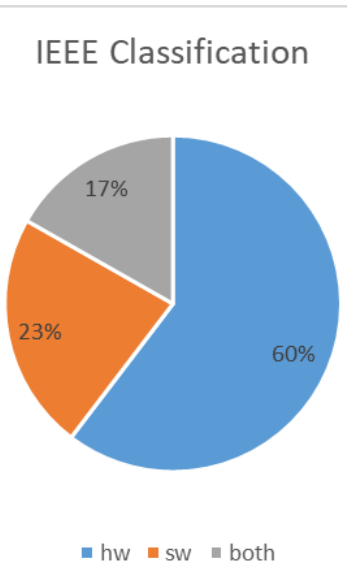

Figure 2. Approximate
Computing IEEE
Classification

In a more detailed view, IEEE classified the articles by "Index Terms", and each one was roughly classified as "software" (sw), "hardware" (hw) or "both" (sw and hw), according to the term. For instance, all keywords related to "circuits" or "energy" were considered as "hardware". On the other hand, the ones that are most probably done only with "software" such as "multimedia" or "artificial intelligence", were classified as software. And other keywords that are mixed were classified as "both", such as "IoT".

Considering these classifications terms, it could be seen that $60 \%$ of the studies are hardware-based, and only $23 \%$ are software-based, the remaining $17 \%$ were classified as both, as shown in Figure 2. The result of the query according to each group of keywords is presented in Table 2 in last subsection. Only groups 2 to 4 will be further analyzed here. The results are summarized in the following tables 3 to 7 .

From Question 2 (Q2) onwards, the analysis was based on articles that deal specifically with software applied to AC. The results were summarized in topics, such as: Focus (Table 3), Hardware (Table 4), Language (Table 5), Results (Table 6) and AC Techniques (Table 7), which were analyzed below.

\section{Conclusion}

This Systematic Review is not yet finished but some conclusions could already be taken, such as the small number of papers regarding AC for weather forecast. Regarding the results of this Review, according to section 2.3, to answering the Questions of Research, considering the analyzed articles, $90.9 \%$ of the articles are related to software (Q1). About the Hardware used, $36.36 \%$ of the studies were done in CPUs, the same percentage remain for FPGAs, and only $9.09 \%$ used GPUs (Q2). Considering the Programming Language, most of them haven't explicitly which were used, but some of them mentioned that $27.27 \%$ used $\mathrm{C} / \mathrm{C}++$ which was the expected result, and 9.09\% mentioned CUDA (Q3). For Q4, which is related to numerical 
models ported, it was not possible to conclude yet since most of the articles do not mention that. And finally, for the last question, regarding the AC techniques used (Q5), most of them, $46.15 \%$ of the articles, used "precision scaling" which include "mixed precision" and any other technique related to numerical precision. Other techniques most used are "load value approximation" and "memoization" with $15.38 \%$ each one.

Table 3 - Focus

\begin{tabular}{|c|c|}
\hline FOCUS & Proportion \\
\hline software & $45.45 \%$ \\
\hline hardware & $9.09 \%$ \\
\hline both & $45.45 \%$ \\
\hline TOTAL & $\mathbf{1 0 0 . 0 0 \%}$ \\
\hline
\end{tabular}

Table 5 - Language

\begin{tabular}{|c|c|}
\hline LANGUAGE & Proportion \\
\hline C / C++ & $27.27 \%$ \\
\hline CUDA & $9.09 \%$ \\
\hline MATLAB & $0.00 \%$ \\
\hline Java & $0.00 \%$ \\
\hline NA & $63.64 \%$ \\
\hline TOTAL & $\mathbf{1 0 0 . 0 0 \%}$ \\
\hline
\end{tabular}

Table 4 - Hardware

\begin{tabular}{|c|c|}
\hline HARDWARE & Proportion \\
\hline CPU & $36.36 \%$ \\
\hline CPU, FPGA & $36.36 \%$ \\
\hline CPU, GPU & $9.09 \%$ \\
\hline CPU, GPU, FPGA & $0.00 \%$ \\
\hline NA & $18.18 \%$ \\
\hline TOTAL & $\mathbf{1 0 0 . 0 0 \%}$ \\
\hline
\end{tabular}

Table 6 - Results

\begin{tabular}{|c|c|}
\hline RESULTS & Proportion \\
\hline acuracy, perfomance & $9.09 \%$ \\
\hline energy saving & $27.27 \%$ \\
\hline performance & $9.09 \%$ \\
\hline $\begin{array}{c}\text { performance, energy } \\
\text { saving }\end{array}$ & $27.27 \%$ \\
\hline NA & $27.27 \%$ \\
\hline TOTAL & $\mathbf{1 0 0 . 0 0 \%}$ \\
\hline
\end{tabular}

Table 7 - AC Techniques

\begin{tabular}{|c|c|}
\hline RESULTS & Proportion \\
\hline Precision scaling & $46.15 \%$ \\
\hline Loop perforation & $7.69 \%$ \\
\hline Load value approximation & $15.38 \%$ \\
\hline Memoization & $15.38 \%$ \\
\hline Task dropping/skipping & $7.69 \%$ \\
\hline Use of neural network & $7.69 \%$ \\
\hline TOTAL & $\mathbf{1 0 0 . 0 0 \%}$ \\
\hline
\end{tabular}

\section{References}

Lim, Y. and Tillmann, U. (1986) "End-to-End Speech Freeze-Out Fractions in a Network with Speech Interpolations”, IEEE Journals , vol. 34, issue 12, pages 1236-1245.

Han, J. and Orshansky, M. (2013) "Approximate computing: An emerging paradigm for energy-efficient design”, IEEE Conferences, pages 1-6.

Li, C., Sengupta, D., Snigdha, F., Xu, W., Hu, J. and Sapatnekar, S. (2017) "Special session: a quantifiable approach to approximate computing”, IEEE Conferences, pages 1-2. 